\title{
Turismo receptivo y crecimiento económico en México: evidencia de largo plazo
}

\author{
Inbound tourism and economic growth in Mexico: Long-run evidence \\ Fernando Sánchez López*
}

Universidad Nacional Autónoma de México

Recibido el 5 de abril de 2018; aceptado el 7 de noviembre de 2018

Disponible en Internet el: 27 de enero de 2020

\section{Resumen}

En este documento se analiza la relación que tienen el PIB real de México y el turismo internacional a través de un modelo ARDL estimado con series trimestrales para el periodo 1993Q1 - 2017Q3. Los resultados señalan la existencia de una relación de largo plazo, y dan evidencia estadística de que el efecto del turismo sobre el PIB es positivo, pero también de que el PIB mexicano es inelástico ante las variaciones en la llegada de turistas internacionales.

Código JEL: C22, L83, Z30, Z32

Palabras Clave: Turismo internacional; Demanda turística; Crecimiento económico; ARDL

\begin{abstract}
In this paper, we analyze the relationship between Mexico's real GDP and international tourism by means of an ARDL model estimated with quarterly series for the period 1993Q1 - 2017Q3. The results

\footnotetext{
*Autor para correspondencia

Correo electrónico fer.sanlop@ciencias.unam.mx (F. Sánchez López).

La revisión por pares es responsabilidad de la Universidad Nacional Autónoma de México.

http://dx.doi.org/10.22201/fca.24488410e.2019.1994

0186- 1042/C 2019 Universidad Nacional Autónoma de México, Facultad de Contaduría y Administración. Este es un artículo Open Access bajo la licencia CC BY-NC-SA (https://creativecommons.org/licenses/by-nc-sa/4.0/)
} 
indicate the existence of a long-run relationship, and provide statistical evidence that international tourism has a positive effect on the GDP; although the GDP is inelastic to the changes in tourist arrivals. JEL code : $\mathrm{C} 22, \mathrm{~L} 83, \mathrm{Z} 30, \mathrm{Z} 32$

Keywords: International tourism; Tourism demand; Economic growth; ARDL

\section{Introducción}

En la actualidad, la industria del turismo es considerada una de las más grandes e importantes del mundo, e incluso algunas estimaciones pronostican que en los próximos años uno de cada diez empleos será creado por dicho sector (Chou, 2013). Además, para diversas naciones el turismo se ha convertido en un verdadero motor de desarrollo sostenible, crecimiento económico y generación de divisas (McCatty y Serju, 2006; Touhami, 2014).

Del mismo modo, el desarrollo del sector turístico impacta la inversión extranjera directa (Ferguson, 2010; Işik, 2015), lo que se refleja ya sea en proyectos privados de gran escala como hoteles y resorts de lujo, o en los destinos de turismo alternativo y de mochileros (Ferguson, 2010).

Debido a lo anterior, al turismo se le atribuyen efectos positivos en la lucha contra la pobreza que, de acuerdo con Ferguson (2010), se pueden resumir en tres puntos cruciales: mejora de las condiciones macroeconómicas a través de la diversificación de la economía y las aportaciones a la balanza de pagos; facilita la expansión empresarial en los países en desarrollo y creación de empleos.

En efecto, el desarrollo del turismo se aprecia como un medio para el crecimiento y el progreso en naciones con alto potencial para atraer turistas, como es el caso de Nigeria (Ezenagu, 2013). En Malí se han puesto en marcha diversos proyectos para fortalecer la conexión entre turismo y seguridad alimentaria (Richardson, 2010). En naciones como Haití, el turismo ha sido elevado a nivel de prioridad por el gobierno, pues se cree que puede ayudar al país a depender menos de la ayuda internacional (Kolbe et al., 2013).

En el caso de México, siguiendo a Girault (2013), el país cuenta con diversas ventajas para explotar su potencial turístico, por ejemplo, un clima generalmente agradable, la gente se percibe como amena y hospitalaria, a pesar de los riesgos que en algunas ocasiones son claramente visibles, y muchas oportunidades para realizar actividades de carácter lúdico o cultural. A lo que se suman sus propiedades inscritas como Patrimonio Mundial, que a la fecha suman 34 en total, 27 de carácter cultural, 6 naturales y una mixta (UNESCO, 2018).

Así pues, durante 2016, México captó poco más de 35 millones de turistas que lo situaron como el número ocho entre los países que mayor cantidad de visitantes internacionales re- 
ciben. Además, en ese mismo año, se colocó en la posición 14 del mundo entre las naciones que más divisas atrajeron gracias al sector turístico (Datatur, 2018).

Con el objetivo de medir el impacto de la llegada de turistas internacionales sobre el crecimiento económico de México, en este trabajo se emplea un Modelo Autorregresivo de Rezagos Distribuidos, ARDL, por sus siglas en inglés, ya que dicha metodología permite probar la existencia de cointegración en el marco de una sola ecuación, e independientemente de si las variables son, o una combinación de ambas, siempre que el orden de integración sea menor a dos, además, es posible determinar el número de rezagos óptimo para cada variable considerada (Nkoro y Uko, 2016).

El modelo se calculó con series trimestrales para el periodo 1993Q1 - 2017Q3, poniendo al logaritmo del Producto Interno Bruto, PIB, real de México, , en función del logaritmo del número de turistas internacionales que arribaron a suelo mexicano, . Los resultados dan evidencia del impacto positivo del turismo en la economía mexicana, y de la existencia de una relación de largo plazo entre las variables en estudio. No obstante, se encuentra que el PIB real es inelástico ante las variaciones en el número de turistas, $\mathrm{y}$, mediante el modelo de corrección de errores, se halla que la convergencia al equilibrio es lenta.

Este estudio se ha dividido en tres partes, sin considerar la presente introducción, la primera corresponde a la revisión de literatura, la segunda se ha divido en dos secciones, primero se presentan los hechos estilizados de la llegada de turistas internacionales a México, y posteriormente algunos indicadores de la relación entre la llegada de viajeros y la economía mexicana, en el tercer apartado se muestran los resultados econométricos. Finalmente se discuten las conclusiones.

\section{Revisión de literatura}

La demanda turística se define, siguiendo a Ascanio (2012), por dos factores, el primero corresponde a los flujos de viajeros que salen de su residencia habitual, y, en segundo lugar, por la distribución de tales corrientes turísticas en los sitios receptores.

Por su parte, Panosso y Lohmann (2012) precisan que la demanda turística es el total de personas participantes en actividades de tipo turístico, la cual se cuantifica a través del número de llegadas o salidas de viajeros, el total de dinero gastado u otros datos estadísticos. Mientras que Cárdenas (1990) señala que la demanda turística es el conjunto de bienes y servicios efectivamente solicitados por el consumidor, y, por lo tanto, abarca todo el mercado actual.

Así pues, siguiendo a Bote (1990) y a Tribe (2011), los principales rubros en que gastan los turistas son: viajes, transporte, alojamiento y alimentación, y otros gastos que no se in- 
cluyen del todo en los paquetes turísticos, por ejemplo, excursiones y compras en general.

Una de las formas más utilizadas para aproximarse al efecto de la demanda turística en las naciones, es medir el impacto del gasto de los turistas sobre el crecimiento económico, pues es de suma importancia para los hacedores de política económica y turística (Chou, 2013), ya que las compras realizadas por los turistas internacionales equivalen a una exportación para la nación receptora (Acerenza, 2006).

En efecto, cualquier venta a un turista extranjero cuenta como una exportación, la diferencia está en que, en el turismo, el consumidor acude al oferente, y no al revés, lo que permite a las pequeñas empresas entrar en la cadena de suministro global (UNCTAD, 2007). Por el contrario, el gasto que realizan los turistas una vez fuera de su país se contabiliza como una importación para la nación emisora (Boullón, 2009; Seetaram, 2010), y, de acuerdo con Sosa (2001), las importaciones son una función del ingreso.

Es así que la demanda turística procedente del exterior tiene como uno de sus más importantes determinantes al ingreso en la nación emisora (Ascanio, 2012; Boer, 2016; Chevillon y Timbeau, 2006; Dahdá, 2003; Panosso y Lohmann, 2012; Stabler et al. 2010), pues los viajes con fines recreacionales se suelen realizar una vez que se han cubierto las necesidades elementales (Ascanio, 2012; Kim et al, 2012; Panosso y Lohmann, 2012). Aunque Ripoll (1986) menciona que un ingreso bajo inhibe los viajes sólo hasta cierto punto, pues el viajero potencial podría obtener un crédito.

Ahora bien, al analizar el impacto que el gasto turístico tendrá sobre la economía de la nación receptora, se debe considerar que el efecto dinamizador dependerá fuertemente de que tan alto sea el contenido en importaciones (Bote, 1990; Hernández, 2004).

Existen diversos estudios cuyos resultados dan evidencia de cointegración entre las ganancias derivadas del turismo, o el gasto turístico, y el crecimiento económico para naciones como Colombia (Such et al., 2009; Brida et al., 2011), España (Balaguer y Cantavella-Jordá, 2002), Grecia (Bayramoğlu y Ari, 2015), México (Brida et al., 2008c), Nepal (Paudyal, 2012), Singapur (Lean et al., 2014), Sri Lanka (Srinivasan et al., 2012), Sudáfrica (Phiri, 2016), Turquía (Akan et al., 2007) y Uruguay (Brida et al., 2008a).

En el caso de los estudios de datos panel, Castro-Nuño et al. (2013) encuentran, haciendo una revisión de distintas estimaciones, que la elasticidad entre el PIB y el turismo es positiva. No obstante, comentan que cuando se usa al turismo como única variable explicativa los coeficientes suelen estar sobrestimados.

En efecto, la estimación por datos panel de Shakouri et al (2017), para una selección de países asiáticos, muestra evidencia de cointegración entre los ingresos generados por el turismo y el crecimiento del PIB per cápita. 
Por el contrario, Ekanayake y Long (2012) encuentran que la elasticidad del PIB con respecto al ingreso generado por el turismo es positiva, pero estadísticamente no significativa. Sin embargo, dada la positividad del efecto, sugieren que los gobiernos de tales naciones se centren en promover la actividad turística como un motor potencial de crecimiento económico.

Otra forma de aproximarse al efecto de la demanda turística en las economías nacionales, ha sido medir el impacto de la llegada de viajeros sobre el crecimiento. Evidencia empírica con resultados favorables a este enfoque ha sido encontrada por Eugenio-Martín et al. (2004), Kum et al. (2015), Lau et al. (2008), Loría et al. (2017) y Phiri (2016).

Al respecto, Phiri (2016) señala que la llegada de turistas internacionales valida la hipótesis de crecimiento económico cuando se utilizan métodos lineales de cointegración, pero no halla tal evidencia al utilizar metodologías no lineales. Mientras que el enfoque de Loría et al. (2017), a través de la Ley de Okun, permite verificar que la llegada de viajeros totales ayuda también a disminuir la tasa de desempleo.

Una observación que se debe tener presente al analizar los resultados de las estimaciones, es que, además de depender del nivel de especialización que la nación en cuestión tenga en turismo, son sensibles a la especificación del modelo, y a la metodología econométrica seleccionada (Pablo-Romero y Molina, 2013).

Del mismo modo, es necesario recordar que para lograr crecimiento económico vía turismo, tan importante es contar con un sector turístico desarrollado, como con una integración adecuada del mismo al resto de las actividades económicas (Brida et al., 2008b). En particular se deben crear encadenamientos productivos con el sector alimentario y las otras ramas proveedoras de servicios, así como procurar que el sector sea una verdadera fuente de empleos, ya que de esta manera el turismo quedará enlazado al combate contra la pobreza (UNCTAD, 2013).

En este sentido, Čerović et al. (2015) encuentran que el turismo tiene diferentes niveles de impacto en las economías, y que está fuertemente ligado a la diversidad y calidad de la oferta, por lo que es necesario adaptarla a las tendencias turísticas internacionales, y, como comentan Altimira y Muñoz (2007), es importante promover el respeto y la conservación del patrimonio turístico para mantener la competitividad del sector.

En adición, se tienen que considerar los factores no estrictamente económicos como la estabilidad social de las regiones que reciben visitantes, pues autores como Boer (2016), Sánchez y Cruz (2015) y Sánchez y Cruz (2017) advierten que la violencia tiene efectos negativos sobre la entrada de viajeros extranjeros. En este sentido, Andrés-Rosales et al. (2018) hallan que la caída del turismo generada por la violencia impacta directamente en el bienestar de los residentes, pues se detiene la creación de empleos temporales, y con ello la obtención de ingresos complementarios, minando los efectos benéficos del turismo. 
Finalmente, en este estudio se analiza el impacto de la llegada de turistas internacionales en la economía mexicana a través de un modelo ARDL estimado con series trimestrales para el periodo 1993Q1 - 2017Q3, que pone como variable dependiente al PIB real y como explicativa al turismo internacional. Los resultados indican que la llegada de viajeros estimula el crecimiento económico, aunque el efecto se puede considerar bajo, de hecho, los resultados señalan que el PIB es inelástico ante las variaciones en la recepción de turistas procedentes del extranjero. Asimismo, el Modelo de Corrección de Errores muestra que la convergencia al equilibrio es lenta.

\section{Análisis descriptivo del turismo receptivo en México}

\section{Llegada de turistas internacionales}

México se ha consolidado como uno de los principales destinos turísticos a nivel global, alcanzando la octava posición entre los países más visitados del mundo durante 2016, además, el único retroceso en la recepción de turistas se dio en 2009 (Datatur, 2018), año de la crisis financiera internacional.

Ahora bien, de acuerdo con el Instituto Nacional de Estadística y Geografía, INEGI, (2018), el turismo internacional se divide en dos categorías: turismo de frontera y turismo de internación, presentando ambos segmentos tendencia positiva durante los últimos años que comprende este estudio (Figura 1).

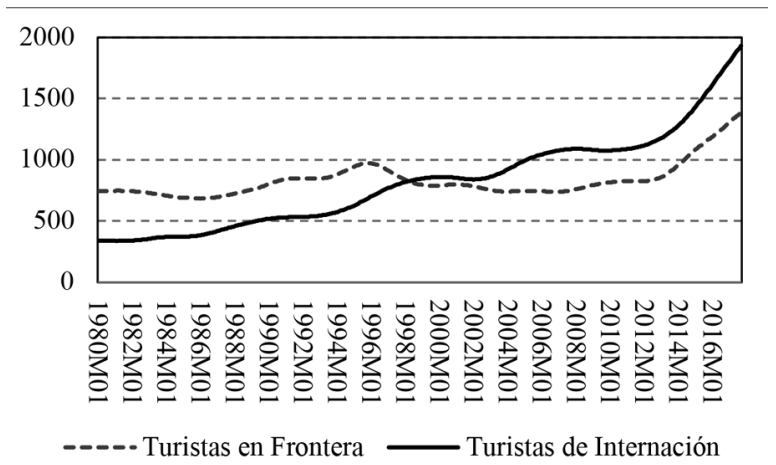

Figura 1. México: tendencias del turismo en frontera y de internación, 1980M1 - 2017M09.

Nota: ${ }^{1 /}$ Miles de personas; ${ }^{2}$ Las tendencias se obtuvieron aplicando el filtro Hodrick-Prescott con $\lambda=14400$ como parámetro de suavizamiento a series previamente desestacionalizadas con el filtro Census X12.

Fuente: Elaboración propia con datos del INEGI (2018). 
La Figura 1 muestra que durante los últimos años el turismo de internación superó al de frontera, lo que es sumamente importante, ya que, por definición, el turismo de internación es aquel que pasa al menos una noche en algún tipo de alojamiento, y el medio de transporte por el que ingresan al país puede ser aéreo o terrestre (Secretaria de Turismo, 2008).

Con respecto al turismo de frontera, es importante notar que las entradas permanecieron casi estancadas durante la década pasada, y es hasta la primera parte de la actual que comenzaron a crecer, alcanzando su mayor nivel durante los últimos meses que abarca este estudio (Figura 1). Los turistas fronterizos acceden a territorio mexicano ya sea a pie, o en automóvil (INEGI, 2018).

El pronunciado ascenso en la llegada de turistas internacionales hacia el final del periodo estudiado coincide con la inestabilidad cambiaria del peso mexicano frente al dólar americano que se presentó en aquellos meses, lo que es congruente con lo expuesto por Chevillon y Timbeau (2006).

\section{Turismo internacional y crecimiento}

A partir del año 2001, el turismo ha representado más de $8 \%$ del PIB nacional mexicano, alcanzando su mayor participación en la economía durante los años de 2008 y 2009, cuando representó $8.82 \%$ y $8.8 \%$ del PIB total real, respectivamente (Figura 2).

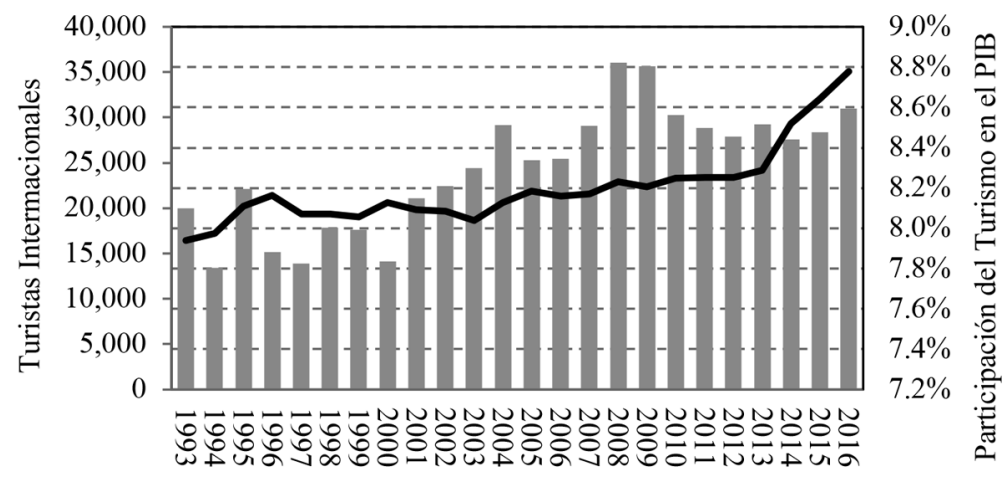

Participación del Turismo en el PIB Nacional —Llegada de Turistas

Figura 2. México: llegada de turistas y participación del turismo en el PIB real, 1993 - 2016

Nota: Los turistas se miden en miles de personas.

Fuente: Elaboración propia con datos del INEGI (2018). 
Un hecho que resalta de la Figura 2 es que los años de mayor participación del turismo en la economía no han sido aquellos en los que se ha recibido mayor número de turistas internacionales, como ocurrió durante 2008 y 2009.

Ahora bien, la Figura 3 da evidencia de la relación positiva que existe entre las tasas de crecimiento del PIB y de la llegada de turistas internacionales.

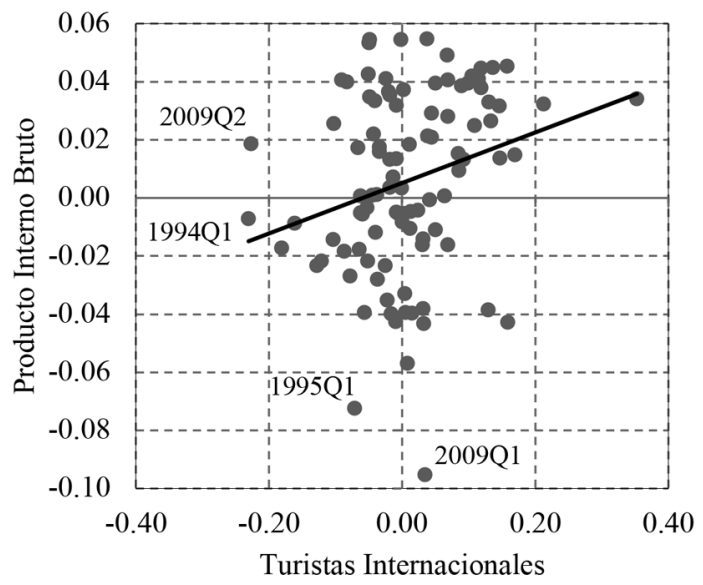

Figura 3. México: tasas de crecimiento del PIB real y de la llegada de turistas, 1993Q2 - 2017Q3 Nota: La gráfica está elaborada con series estacionarias (Tabla A1). Fuente: Elaboración propia con datos del INEGI (2018).

Durante los trimestres 1995Q1 y 2009Q1, la economía mexicana sufrió fuertes caídas, consecuencia ambas de crisis económicas. Luego, durante el segundo trimestre de 2009, se dio una fuerte caída en el turismo receptivo que da evidencia de que el sector es muy sensible a los periodos de crisis, como señala Álvarez (1996).

Otra severa caída en la recepción de turistas internacionales se dio durante el primer trimestre de 1994, que coincide con la toma por las armas, la madrugada del $1^{\circ}$ de enero de aquel año, de cinco cabeceras municipales del estado de Chiapas, entre ellas la de San Cristóbal de las Casas, por parte del Ejército Zapatista de Liberación Nacional. A lo que el gobierno respondió enviando al ejército para sofocar la rebelión, lo que provocó enfrentamientos que se prolongarían durante 11 días (Estrada, 2011).

Para respaldar la relación entre las tasas de crecimiento del turismo y el PIB real presentada en la Figura 3, se aplicó la prueba de causalidad en el sentido de Granger, cuyos resultados se resumen en la Tabla 1. 
Tabla 1

Prueba de causalidad en el sentido de Granger

\begin{tabular}{lll}
\hline Muestra: 1993Q2 2017Q3 & & \\
\hline Rezagos: 1 & & \\
\hline Hipótesis Nula: & Estadístico F & Valor P \\
\hline No Granger causa a & 6.82421 & 0.0105 \\
No Granger causa a & 0.70520 & 0.4032 \\
\hline
\end{tabular}

Nota: La prueba se llevó a cabo con series estacionarias (Tabla A1).

Fuente: Elaboración propia con datos del INEGI (2018).

La información del Tabla 1 señala que existe una relación estadísticamente significativa de la llegada de turistas sobre el crecimiento económico, pero no al revés, lo que es congruente con lo hallado por Loría et al. (2017) para la llegada de viajeros internacionales totales a México.

\section{Resultados Econométricos}

En esta sección, a partir de un modelo ARDL que tiene como variable dependiente al logaritmo natural del PIB real mexicano, y como variable explicativa al logaritmo natural del turismo receptivo, , se estudia el impacto de la llegada de turistas internacionales sobre el PIB con series trimestrales para el periodo 1993Q1 - 2017Q3 (Figura 4).
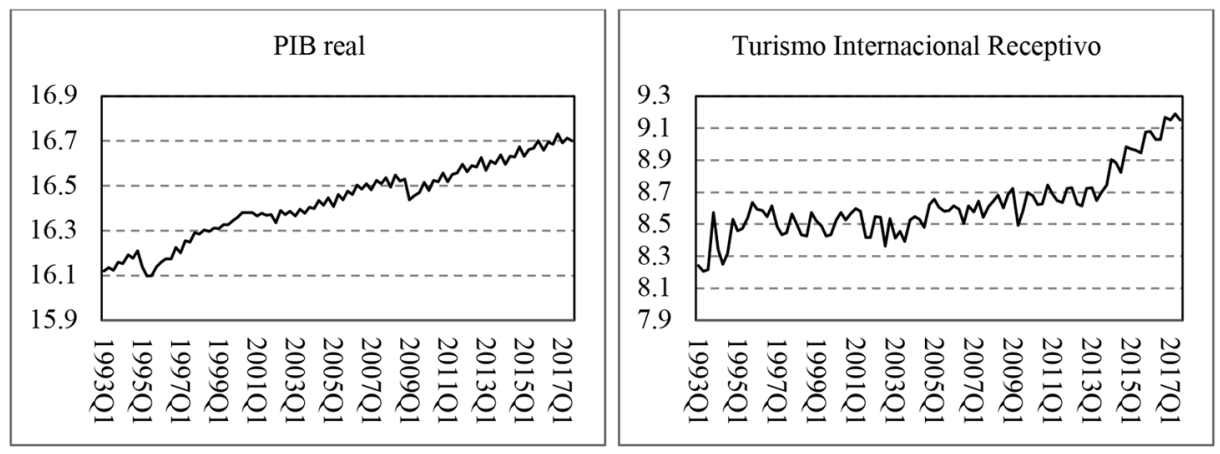

Figura 4. Variables que intervienen en el modelo ARDL.

Nota: Series en logaritmos naturales.

Fuente: Elaboración propia con datos del INEGI (2018). 
Se ha verificado mediante las pruebas de raíz unitaria Augmented Dickey-Fuller, ADF, Phillips-Perron, PP y Kwiatkowski-Phillips-Schmidt-Shin, KPSS, que las series y son de orden de integración uno, es decir (Tabla A1).

El modelo también contiene, como variables estáticas, una constante, y una variable de intervención, , que ayuda a corregir el cambio estructural en el modelo y a simular adecuadamente aquellos periodos en que la serie del PIB real presenta fuertes quiebres: 1995Q1, 1995Q2, 1996Q1, 2002Q2, 2008Q2 y 2009Q1.

Para determinar el número de rezagos en el modelo se utilizó el criterio de Akaike, permitiéndose un máximo de seis retardos en ambas variables. El criterio indica que la mejor estimación es un ARDL $(5,2)$ (Figura A1). Los resultados se resumen en la Tabla 2.

Tabla 2

Modelo ARDL $(5,2)$

\begin{tabular}{lll}
\hline \multicolumn{2}{l}{ Variable dependiente: $\ln Y_{t}$} & \\
\hline Variable & Coeficientes & Valor P \\
\hline $\ln Y_{t-1}$ & 0.8298 & $0.0000^{* * *}$ \\
$\ln Y_{t-2}$ & 0.2077 & $0.0096^{* * *}$ \\
& & \\
$\ln Y_{t-3}$ & -0.3137 & $0.0001^{* * *}$ \\
$\ln Y_{t-4}$ & 0.6300 & $0.0000^{* * *}$ \\
$\ln Y_{t-5}$ & -0.3922 & $0.0000^{* * *}$ \\
$\ln T_{t}$ & 0.0153 & 0.4372 \\
$\ln T_{t-1}$ & -0.0554 & $0.0250^{* *}$ \\
$\ln T_{t-2}$ & 0.0680 & $0.0007^{* * *}$ \\
$d_{t}$ & 0.0437 & $0.0000^{* * *}$ \\
$C$ & 0.3949 & $0.0232^{* *}$ \\
\hline
\end{tabular}

Nota: Los superíndices $* *$ y $* * *$ expresan significancia al $5 \%$ y $1 \%$, respectivamente.

Fuente: Elaboración propia con datos del INEGI (2018).

Se verificó que el modelo satisficiera apropiadamente las pruebas de correcta especificación al 5\% de significancia, así como las pruebas de bondad de ajuste. ${ }^{1}$ De la misma manera, se corroboró la estabilidad del ARDL mediante las pruebas CUSUM y CUSUM Cuadrado (Figura A2).

\footnotetext{
${ }^{1}$ Jarque-Bera $=1.5718(0.4557)$; Breusch-Godfrey LM (12) = $14.6526(0.2610)$; White (n. c. $)=5.5156(0.7872)$; White $(\mathrm{c})=$ 22.5946 (0.6012); ARCH LM (12) = 18.5517 (0.0999); RESET Ramsey = 0.0167 (0.8973); $\mathrm{R}^{2}=0.99337$; $\mathrm{R}^{2}$-ajustada $=0.99266$; Estadístico $\mathrm{F}=1399.375$ (0.0000).
} 
Se realizó también la prueba de punto de quiebre desconocido de Quandt-Andrews, hallándose que en presencia de la variable de intervención es posible rechazar estrictamente la hipótesis de cambio estructural, mientras que en ausencia de dicha variable el modelo adolece de tal eventualidad (Tabla A2).

Como última prueba de correcta especificación al modelo ARDL, se constató que simulara adecuadamente la variable dependiente (Figura 5).

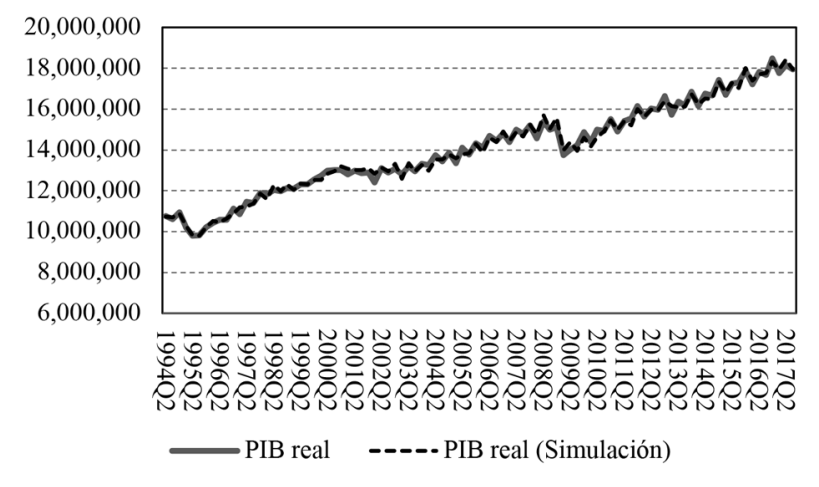

Figura 5. Simulación histórica del modelo ARDL

Nota: Para simular el PIB real se utilizó el algoritmo de Broyden.

Fuente: Elaboración propia a partir del modelo ARDL (Tabla2) y datos del INEGI (2018).

Como se puede apreciar en la Figura 5, el modelo ARDL recoge adecuadamente los principales quiebres en la serie del PIB real.

Dado que el modelo ARDL satisface las pruebas de correcta especificación, y que las pruebas de raíz unitaria señalan que las series usadas son, se aplicó la prueba de límites, con la que se corrobora la existencia de una relación de largo plazo entre el turismo receptivo y el PIB real (Tabla 3).

Tabla 3

Prueba de límites

\begin{tabular}{lcc}
\hline & Valores Críticos & \\
\cline { 2 - 3 } Estadístico F = 6.573368 & Límite Inferior & Límite Superior \\
& $\mathrm{I}(0)$ & $\mathrm{I}(1)$ \\
\hline Nivel de Significancia 10\% & 3.02 & 3.51 \\
Nivel de Significancia 5\% & 3.62 & 4.16 \\
Nivel de Significancia 1\% & 4.94 & 5.58 \\
\hline
\end{tabular}

Nota: Hipótesis nula: No existe relación de largo plazo.

Fuente: Elaboración propia. 
En la Tabla 3 se aprecia que el valor del estadístico F excede el valor crítico del límite al $1 \%$ de significancia, lo que permite rechazar estrictamente la hipótesis nula de no existencia de una relación de largo plazo entre las variables en estudio, por lo que tiene sentido estimar los coeficientes de largo plazo (Tabla 4).

Tabla 4

Coeficientes de largo plazo

\begin{tabular}{ccc}
\hline \multicolumn{3}{c}{ Variable Dependiente: $1 \mathrm{n} Y$} \\
\hline Variable & Coeficientes & \multicolumn{1}{l}{ Valor P } \\
\hline$C$ & 10.3061 & $0.0000^{* * *}$ \\
$\ln T$ & 0.7292 & $0.0006^{* * *}$ \\
$d$ & 1.1418 & $0.0140^{* *}$ \\
\hline
\end{tabular}

Nota: Los superíndices $* *$ y $* * *$ expresan la significancia al $5 \%$ y $1 \%$ respectivamente. Fuente: Elaboración propia a partir del Modelo ARDL (Tabla 2).

Luego, se presenta el Modelo de Corrección de Errores, MCE, (Tabla 5), donde el término de corrección del error, , está definido como se muestra en (1)

$$
C E_{t}=\ln Y_{t}-\left(0.72921 \mathrm{n} T_{t}+1.1418 d_{t}+10.3061\right.
$$

El MCE señala que el coeficiente asociado a es negativo y estadísticamente significativo, como requiere la metodología de los modelos ARDL, ya que, siguiendo a Nkoro y Uko (2016), tales características indican convergencia, por lo que tiene sentido afirmar que existe una relación de largo plazo.

Así pues, el coeficiente correspondiente a $C E_{t-1}$ exhibe que $4.2 \%$ del desequilibrio del PIB real del periodo previo se ajusta en el periodo en curso (Tabla 5). 
Tabla 5

Modelo de corrección de errores

\begin{tabular}{ccc}
\hline \multicolumn{2}{c}{ Variable dependiente: $\Delta \ln Y_{t}$} & \\
\hline Variable & Coeficientes & Valor P \\
\hline$\Delta \ln Y_{t-1}$ & -0.1769 & $0.0339^{* *}$ \\
$\Delta \ln Y_{t-2}$ & 0.0495 & 0.4552 \\
$\Delta \ln Y_{t-3}$ & -0.2348 & $0.0002^{* * *}$ \\
$\Delta \ln Y_{t-4}$ & 0.3813 & $0.0000^{* * *}$ \\
$\Delta \ln Y_{t}$ & 0.0195 & 0.3107 \\
$\Delta \ln Y_{t-1}$ & -0.0672 & $0.0003^{* * *}$ \\
$\Delta d_{t}$ & 0.0427 & $0.0000^{* * *}$ \\
$C E_{t-1}$ & -0.0420 & $0.0000^{* * *}$ \\
\hline
\end{tabular}

Nota: Los superíndices $* *$ y $* * *$ expresan significancia al $5 \%$ y $1 \%$, respectivamente.

Fuente: Elaboración propia a partir del modelo ARDL (Tabla 2).

Con base en los resultados de las pruebas de correcta especificación, la prueba de límites y del MCE, tiene sentido interpretar los coeficientes de largo plazo (Tabla 4).

La ecuación de largo plazo indica que el efecto de la llegada de turistas internacionales sobre el PIB real es positivo, ya que por cada $1 \%$ que crece la llegada de turistas, el PIB aumenta en $0.72 \%$. Sin embargo, tal resultado implica que el crecimiento del PIB real es menos que proporcional ante los cambios en la llegada de turistas, o, en otras palabras, que el PIB es inelástico ante las variaciones en el número de turistas internacionales.

Lo anterior muestra que la llegada de turistas, si bien tiene un efecto positivo y estadísticamente significativo en la economía, también lo es que tiene un impacto que se puede considerar bajo. Además, el MCE revela que la convergencia al equilibrio es lenta.

Se debe tomar en cuenta que la variable empleada para medir la demanda turística es la llegada de turistas internacionales, pues no todos los estratos generan el mismo impacto en el territorio al que llegan. Efectivamente, las personas que conforman los segmentos de turismo familiar y de amigos, religioso y estudiantil, por lo general realizan desembolsos por debajo de las otras clasificaciones (Cárdenas, 1990).

Por último, si bien la captación de turistas tiene un efecto positivo para la economía, se debe procurar, como recomienda Boullón (2009), que los visitantes sientan deseos de realizar desembolsos más cuantiosos, lo que podría lograrse poniendo en marcha una estrategia para aumentar el tiempo de permanencia en el país, pues en el turismo receptivo un día más implica, al menos, una pernoctación extra, y mayor gasto en alimentación. 


\section{Conclusión}

En este documento se ha estudiado la relación entre el PIB real de México y la entrada de turistas internacionales, encontrándose, a través de un modelo ARDL, estimado con series trimestrales para el periodo 1993Q1 - 2017Q3, que la llegada de turistas internacionales alienta el crecimiento económico. Asimismo, mediante la prueba de límites, se ha hallado la existencia de una relación de largo plazo entre las variables en estudio, que se corrobora con el signo negativo del coeficiente del término de corrección del error.

No obstante, el coeficiente asociado al turismo internacional en la ecuación de largo plazo muestra que el crecimiento del PIB real se puede considerar bajo cuando aumentan las entradas de turistas. Más precisamente, la ecuación de largo plazo muestra que el PIB real crece a penas en $0.72 \%$ cuando la llegada de turistas aumenta en un punto porcentual.

Lo arriba expuesto indica que, durante el periodo estudiado, el PIB mexicano ha sido inelástico ante los cambios en la llegada a suelo mexicano de extranjeros y residentes en el exterior con fines turísticos.

De la misma manera, en el MCE, el coeficiente correspondiente a señala que las variables convergen lentamente al equilibrio. Más específicamente, que sólo $4.2 \%$ del desequilibrio del PIB real en el periodo previo se ajusta en el periodo actual.

Al utilizar tasas de crecimiento, la prueba de causalidad en el sentido de Granger da evidencia de que el turismo tiene un impacto estadísticamente significativo sobre el PIB real, mostrando que las variables en estudio se relacionan también en el corto plazo. Además, la pendiente de la recta ajustada por mínimos cuadros en la gráfica de dispersión indica que la relación PIB - turismo internacional es positiva (Figura 3).

Así pues, si bien la llegada de turistas internacionales estimula la economía en su conjunto, se deben buscar estrategias que lo hagan un mejor propulsor del crecimiento económico. En este sentido, la política turística debe promover los estratos turísticos que mayor impacto económico tienen, en particular se debe fomentar la llegada de aquellos viajeros que hacen uso de los servicios de hospedaje, dado su mayor desembolso. Asimismo, es necesario promover el deseo de los visitantes por gastar sumas más cuantiosas durante su estancia.

Desde luego, lo anterior no significa que se descuiden segmentos tan importantes como el turismo estudiantil, ya que, a través de una buena experiencia durante su estadía, podrían volverse verdaderos promotores del país una vez que hayan vuelto a sus naciones de residencia, como señala Cárdenas (1990). 
Asimismo, se debe procurar la conservación del patrimonio turístico nacional, y, cuando corresponda, su actualización y modernización, ya que la demanda de turismo responde ante la calidad y diversidad de la oferta.

Finalmente, será necesario poner atención en factores como la seguridad que se brinda en los sitios turísticos, no solamente para conservar o aumentar el nivel de demanda turística, sino por la calidad de vida de los residentes, tanto de aquellos cuyos ingresos están en función de los turistas, como de los no dependientes.

\section{Referencias}

Acerenza, M. A. (2006). Efectos económicos, socioculturales y ambientales del turismo. Trillas, México.

Akan, Y., Arslan, I. e Işik, C. (2007). The impact of tourism on economic growth: The case of Turkey. Journal of Tourism 9, 1-24. Disponible en: https://ssrn.com/abstract=1346918 (Consultado: 18/02/2018).

Altimira, R. y Muñoz, X. (2007). El turismo como motor de crecimiento económico. Anuario Jurídico y Económico Escurialense 40, 677-710. Disponible en: https://dialnet.unirioja.es/servlet/articulo?codigo=2267966 (Consultado: 19/02/2018).

Álvarez, P. (1996). La relación de los servicios y el turismo con el sector externo en México. Comercio Exterior 46 (2), 148-157. Disponible en: http://revistas.bancomext.gob.mx/rce/magazines/310/8/RCE8.pdf (Consultado: 20/02/2018).

Andrés-Rosales, R., Sánchez-Mitre, L. A. y Cruz, J. N. (2018). Insecurity and its impact on tourism in Guerrero: a spatial approach, 1999-2014. Revista de Relaciones Internacionales, Estrategia y Seguridad 13 (1), $147-162$. https://doi.org/10.18359/ries.2977.

Ascanio, A. (2012). Teoría del turismo. Trillas, México.

Balaguer, J. y Cantavella-Jordá, M. (2002). Tourism as a long-run economic factor: The case of Spain. Applied Economics 34, 877-884. https://doi.org/10.1080/00036840110058923.

Bayramoğlu, T. y Ari, Y. O. (2015). The relationship between tourism and economic growth in Greece economy: A time series analysis. Computational Methods in Social Sciences 3 (1), 89-93. Disponible en: http://cmss. univnt.ro/wp-content/uploads/vol/split/vol_III_issue_1/CMSS_vol_III_issue_1_art.009.pdf (Consultado: $18 / 02 / 2018)$.

Boer, J. (2016). Le tourisme: un moteur de l'économie mondiale. Cahiers Français (393), 8-13. Disponible en: http://www.ladocumentationfrancaise.fr/var/storage/libris/3303330403938/3303330403938_EX.pdf (Consultado: 12/03/2018).

Bote, V. (1990). Planificación económica del turismo: de una estrategia masiva a una artesanal. Trillas, México.

Boullón, R. C. (2009). Las actividades turísticas y recreacionales: el hombre como protagonista. Trillas, México.

Brida, J. G., Lanzilotta, B. y Risso, W. A. (2008a). Turismo y crecimiento económico: el caso de Uruguay. Pasos 6 (3), 481-492. https://doi.org/10.25145/j.pasos.2008.06.036.

Brida, J. G., Pereyra, J. S., Such, M. J. y Zapata, S. (2008b). La contribución del turismo al crecimiento económico. Cuadernos de Turismo (22), 35-46. Disponible en: http://revistas.um.es/turismo/article/view/47931 (Consultado:18/02/2018).

Brida, J. G., Sánchez, E. J. y Risso, W. A. (2008c). Tourism's impact on long-run Mexican economic growth. Economics Bulletin 3 (21), 1-8. https://doi.org/10.2139/ssrn.1076225.

Brida, J. G., Monterubbianesi, P. D. y Zapata-Aguirre, S. (2011). Impactos del turismo sobre el crecimiento económico y el desarrollo. El caso de los principales destinos turísticos de Colombia. Pasos 9 (2), $291-303$. https://doi.org/10.25145/j.pasos.2011.09.026. 
Cárdenas, F. (1990). Comercialización del turismo: determinación y análisis de mercados. Trillas, México.

Castro-Nuño, M., Molina-Toucedo, J. A. y Pablo-Romero, M. P. (2013). Tourism and GDP: A meta-analysis of panel data studies. Journal of Travel Research 52(6), 745-758. https://doi.org/10.1177/0047287513478500.

Čerović, S., Knežević, M., Matović, V. y Brdar, I. (2015). The contribution of tourism industry on the GDP growth of Western Balkan countries. Industrija 43(3), 159-170. https://dx.doi.org/10.5937/industrija43-9002 (Consultado: 19/02/2019).

Chevillon, G. y Timbeau, X. (2006). L'impact du taux de change sur le tourisme en France. Revue de l'OFCE (98), 167-181. https://dx.doi.org/10.3917/reof.098.0167.

Chou, M. C. (2013). Does tourism development promote economic growth in transition countries? A panel data analysis. Economic Modelling 33, 226-232. http://dx.doi.org/10.1016/j.econmod.2013.04.024.

Dahdá, J. (2003). Elementos de turismo: economía, alimentos y bebidas, líneas aéreas, hotelería, relaciones públicas. Trillas, México.

Datatur (2018). Estadísticas básicas del sector turismo: ranking mundial del turismo internacional. Secretaria de Turismo. Disponible en: http://www.datatur.sectur.gob.mx/SitePages/RankingOMT.aspx (Consultado: 25/03/2018)

Ekanayake, E. M. y Long, A. E. (2012). Tourism development and economic growth in developing countries. The International Journal of Business and Finance Research 6 (1), 51-63. Disponible en: https://ssrn.com/ abstract=1948704 (Consultado: 21/03/2018).

Estrada, M. (2011). El levantamiento zapatista de 1994. Arqueología Mexicana 19 (111), 60-63. Disponible en: https://arqueologiamexicana.mx/mexico-antiguo/el-levantamiento-zapatista-de-1994 (Consultado: 21/03/2018).

Eugenio-Martín, J. L., Martín, N. y Scarpa, R. (2004).Tourism and economic growth in Latin American countries: a panel data approach. Nota di Lavoro 26, Fondazione Eni Enrico Mattei.

Ezenagu, N. (2013). Tourism a viable path for wealth creation in Nigeria: An analysis of Awka Metropolis. International Journal of Sciences and Research 2 (9), 298-305. Disponible en: https://www.academia.edu/4687218/ Tourism_a_Viable_Path_for_Wealth_Creation_in_Nigeria_An_Analysis_of_Awka_Metropolis (Consultado: 20/03/2018).

Ferguson, L. (2010). Tourism as a development strategy in Central America: Exploring the impact on women's lives. CAWN Briefing Paper, Central America Women's Network.

Girault, C. (2013). Mexique: les atouts d'un Grand du tourisme international. Rayonnement du CNRS (62), 26-35. Hernández, R. (2004). Impacto económico del turismo: el papel de las importaciones como fugas del modelo. ICE, Revista de Economía (817), 23-34. Disponible en: http://www.revistasice.com/CachePDF/ICE_817_2334_B81C35A65AD11B8D459C2D981F9A6641.pdf (Consultado: 20/03/2018).

Instituto Nacional de Estadística y Geografía (2018). Banco de Información Económica.. Disponible en: http:// www.inegi.org.mx/sistemas/bie/ (Consultado: 02/02/2018).

Işik, C. (2015). Foreign direct investment in tourism: Panel data analysis of D7 countries. Athens Journal of Tourism 2(2), 93-103. https://doi.org/10.30958/ajt.2-2-2.

Kim, H., Park, J., Lee, S. y Jang, S. (2012). Do expectations of future wealth increase outbound tourism? Evidence from Korea. Tourism Management 33, 1141-1147. https://doi.org/10.1016/j.tourman.2011.11.017.

Kolbe, A. R., Brookes, K. y Muggah, R. (2013). Is tourism Haiti's magic bullet? An empirical treatment of Haiti's tourism potential. Strategic Note 9, Igarapé Institute.

Kum, H., Aslan, A. y Gungor, M. (2015). Tourism and economic growth: the case of Next-11 countries. International Journal of Economics and Financial Issues 5 (4), 1075-1081. Disponible en: www.econjournals.com (Consultado: 27/02/2018).

Lau, E., Oh, S. y Hu, S. (2008). Tourist arrivals and economic growth in Sarawak. MPRA Paper No. 9888, Munich Personal RePEc Archive. 
Lean, H. H., Chong, S. H. y Hooy, C. (2014). Tourism and economic growth: Comparing Malaysia and Singapore. International Journal of Economics and Management 8(1), 139-157. Disponible en: http://econ.upm.edu.my/ ijem/vol8no1/bab08.pdf (Consultado: 19/02/2018).

Loría, E. G., Sánchez, F. y Salas, E. (2017). Efectos de la llegada de viajeros internacionales en el desempleo y el crecimiento económico en México, 2000.2-2015.2. El Periplo Sustentable (32), 1-24. Disponible en: https:// rperiplo.uaemex.mx/article/view/4877 (Consultado: 20/02/2018).

McCatty, M. y Serju, P. (2006). Tourism, economic growth \& employment. Working Paper, Bank of Jamaica.

Nkoro, E. y Uko, A. K. (2016). Autoregressive Distributed Lag (ARDL) cointegration technique: application and interpretation. Journal of Statistical and Econometric Methods 5 (4), 63-91. Disponible en: http://www.scienpress.com/Upload/JSEM/Vol\%205_4_3.pdf (Consultado: 18/02/2019).

Pablo-Romero, M. P. y Molina, J. A. (2013). Tourism and economic growth: A review of empirical literature. Tourism Management Perspective 8, 28-41. https://doi.org/10.1016/j.tmp.2013.05.006.

Panosso, A. y Lohmann, G. (2012). Teoría del turismo: conceptos, modelos y sistemas. Trillas, México.

Paudyal, S. (2012). Does tourism really matters for economic growth? Evidence from Nepal. NRB Economic Review 24(1), 48-66. Disponible en: https://www.nrb.org.np/ecorev/pdffiles/vol24-1_art4.pdf (Consultado: $18 / 02 / 2018)$.

Phiri, A. (2016). Tourism and economic growth in South Africa: Evidence from linear and nonlinear cointegration frameworks. Managing Global Transitions 14(1), 31-53. Disponible en: http://www.fm-kp.si/zalozba/ ISSN/1581-6311/14_31-53.pdf. (Consultado: 19/02/2018).

Richardson, R. B. (2010). The contribution of tourism to economic growth and food security. USAID from the American People / Mali, Office of Economic Growth. Disponible en: https://ideas.repec.org/p/ags/midcwp/97140. html (Consultado: 20/03/2018).

Ripoll, G. (1986). Turismo popular: inversiones rentables. Trillas, México.

Sánchez, F. y Cruz, J. N. (2015). Efecto de los secuestros en la recepción de excursionistas en crucero: un estudio para México, 1997.1 - 2014.3. Ponencia. XX Congreso Internacional de Contaduría, Administración e Informática. Universidad Nacional Autónoma de México.

Sánchez, F. y Cruz, J. N. (2017). Excursionismo internacional y secuestros en la frontera de México, 1997-2016. Revista de Relaciones Internacionales, Estrategia y Seguridad 12 (1), 237-266. http://dx.doi.org/10.18359/ ries.2471.

Secretaria de Turismo (2008) Visitantes internacionales hacia México, el turismo de internación 2008. Disponible en: http://www.datatur.sectur.gob.mx/Documentos\%20Publicaciones/turint2008.pdf (Consultado: 18/02/2018).

Seetaram, N. (2010). A study of outbound tourism from Australia. Discussion Paper 47/10. Monash University.

Shakouri, B., Yazdi, S. K., Nategian, N. y Shikhrezaei, N. (2017). International tourism and economic growth and trade: variance decomposition analysis. Journal of Tourism \& Hospitality 6 (3), 1-11. https://doi. org/10.4172/2167-0269.1000286.

Sosa, S. W. (2001). Modelos macroeconómicos: de los "clásicos" a la macroeconomía de las economías periféricas. Tlaxcallan, México.

Srinivasan, P., Kuman, S. y Ganesh, L. (2012). Tourism and economic growth in Sri Lanka: an ARDL bounds testing approach. The Romanian Economic Journal 15 (45), 211-226. Disponible en: http://www.rejournal.eu/ article/tourism-and-economic-growth-sri-lanka-ardl-bounds-testing-approach (Consultado: 18/02/2018).

Stabler, M. J., Papatheodorou, A. y Sinclair, T. (2010). The economics of tourism. Routledge, New York. http:// dx.doi.org/10.4324/9780203864272.

Such, J. M., Zapata, S., Risso, W. A., Brida, J. G. y Pereyra, J. S. (2009). Turismo y crecimiento económico: un análisis empírico de Colombia. Estudios y Perspectivas en Turismo 18 (1), 21-35. Disponible en: http://www. redalyc.org/articulo.oa?id=180714242002 (Consultado: 18/02/2018). 
Touhami, L. (2014). L'importance économique et sociale du tourisme mondial et développement durable. Revista de Estudios Fronterizos del Estrecho de Gibraltar (1), 1-18. Disponible en: https://dialnet.unirioja.es/descarga/ articulo/4830593.pdf. (Consultado: 18/02/2018).

Tribe, J. (2011). The economics of recreation, leisure and tourism. Routledge, London. http://dx.doi. org/10.4324/9780080890517.

UNCTAD (2007). FDI in tourism: the development dimension. New York \& Geneva, United Nations. Disponible en: http://unctad.org/en/Docs/iteiia20075_en.pdf (Consultado: 18/02/2018).

UNCTAD (2013). Sustainable tourism: Contribution to economic growth and sustainable development. Issues note prepared by the UNCTAD secretariat. Disponible en: http://unctad.org/meetings/en/SessionalDocuments/ ciem5d2_en.pdf (Consultado: 20/03/2018).

UNESCO (2018). Mexico: Properties inscribed on the World Heritage List. 18 de marzo de 2018. Disponible en: https://whc.unesco.org/en/statesparties/mx (Consultado: 19/02/2018).

\section{Anexo}

\section{Pruebas de raíz unitaria y correcta especificación}

Tabla A1

Pruebas de raíz unitaria, 1993Q1 - 2017Q3

\begin{tabular}{lllllllll}
\hline \multirow{2}{*}{ Series } & ADF & \multicolumn{9}{c}{ PP } & \multicolumn{3}{c}{ KPSS } \\
\cline { 2 - 8 } & A & B & C & A & B & C & A & B \\
\hline $\ln T$ & 0.285 & 1.738 & 1.997 & $-3.683^{*}$ & -2.003 & 2.277 & 0.230 & 1.081 \\
$1 \mathrm{n} Y$ & -3.318 & -2.041 & 3.789 & $-5.213^{*}$ & -0.908 & 4.575 & 0.147 & 1.299 \\
$\Delta \ln T$ & $-12.75^{*}$ & $-5.219^{*}$ & $-4.752^{*}$ & $-16.75^{*}$ & $-16.21 *$ & $-13.22^{*}$ & $0.139^{*}$ & $0.176^{*}$ \\
$\Delta \ln T$ & $-4.290^{*}$ & $-4.314^{*}$ & $-3.434^{*}$ & $-20.70^{*}$ & $-20.77^{*}$ & $-18.40^{*}$ & $0.074^{*}$ & $0.104^{*}$ \\
\hline
\end{tabular}

Notas: ${ }^{1 / A}$.- Pruebas con constante y tendencia, B.- Pruebas sólo con constante, C.- Pruebas sin constante ni tendencia. $2 / *$ Rechazan la hipótesis de raíz unitaria al 5\% de significancia.

Fuente: Elaboración propia con datos del INEGI (2018).

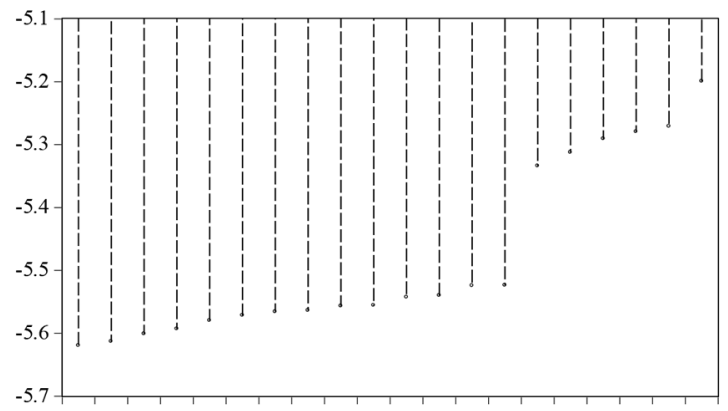

Figura A1. Criterio de Akaike para la selección de rezagos del modelo ARDL

Nota: La figura muestra los mejores 20 modelos de acuerdo al criterio de Akaike.

Fuente: Elaboración propia. 

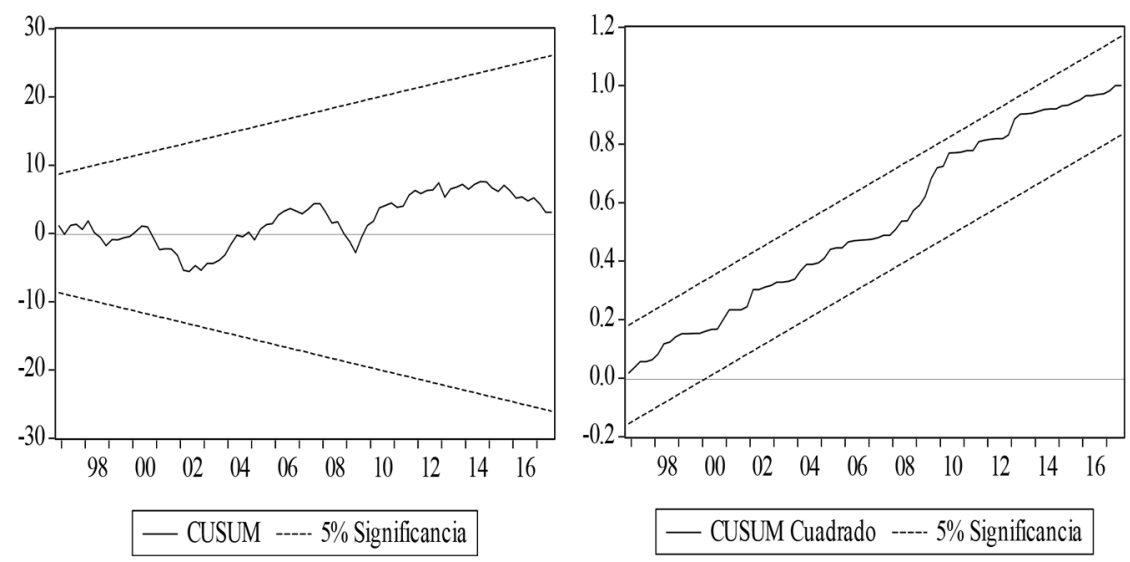

Figura A2. Pruebas de Estabilidad CUSUM y CUSUM Cuadrado.

Fuente: Elaboración propia.

\section{Pruebas de Cambio Estructural}

Se presenta la prueba de Quandt-Andrews de punto de quiebre desconocido, la cual muestra que el modelo adolece de cambio estructural en ausencia de la variable de intervención, lo que se corrige añadiendo la dummy como una variable exógena en el modelo ARDL.

Tabla A2

Prueba de Punto de quiebre desconocido de Quandt-Andrews

\begin{tabular}{lllll}
\hline \multirow{2}{*}{ Prueba } & \multicolumn{3}{c}{ Sin dummy } & \multicolumn{2}{c}{ Con dummy } \\
\cline { 2 - 5 } & Estadístico & Valor P & Estadístico & Valor P \\
\hline Maximum LR F-statistic (2009Q3) & 2.9659 & 0.0319 & 2.3118 & 0.1478 \\
Maximum Wald F-statistic (2009Q3) & 26.6932 & 0.0319 & 23.118 & 0.1478 \\
Exp LR F-statistic & 0.7789 & 0.2275 & 0.5485 & 0.6183 \\
Exp Wald F-statistic & 10.5697 & 0.0208 & 7.6857 & 0.2292 \\
Ave LR F-statistic & 1.4150 & 0.0999 & 1.0678 & 0.3587 \\
Ave Wald F-statistic & 12.7356 & 0.0999 & 10.6783 & 0.3587 \\
\hline
\end{tabular}

Nota: Muestra de la ecuación: 1994Q2-2017Q3; Muestra de la prueba: 1998Q1-2014Q1; 15\% de datos omitidos; Número de quiebres comparados: 65.

Fuente: Elaboración propia con base en el modelo ARDL. 